secreted proteins, and when NF- $\kappa \mathrm{B}$ was inhibited, the cellular secretions no longer promoted metastasis.

Genes Dev. doi:10.1101/ gad.625811 (2011)

\section{EVOLUTION}

\section{Male harassment can doom species}

The males of many species demonstrate behaviours that are harmful to females. Some, for example, can physically damage females during courting or mating. Daniel Rankin at the University of Zurich in Switzerland and his team now demonstrate that such sexual conflict can lead to a 'tragedy of the commons'.

Their models suggest that the evolution of male harassment of females during mate competition can lead to a downward spiral of fewer surviving females; these females are themselves less likely to survive because they receive even more damaging attention from males. Conversely, if female resistance to damaging behaviour evolves, making harassment too costly for males, this can prevent a species from dying out.

Am. Nat. 177, 780-791 (2011)

\section{CELLULAR BIOLOGY}

\section{A microscopist's litmus test}

Intracellular $\mathrm{pH}$ levels can now be measured with a protein that glows red.

The red fluorescent protein has been dubbed pHRed by its designers, Gary Yellen and his colleagues at Harvard Medical School in Boston, Massachusetts. Lightdetecting molecules of pHRed are preferentially excited by light at a wavelength of either 440 nanometres in basic conditions or 585 nanometres in acidic conditions, and the excitation ratio of the two gives a read-out of $\mathrm{pH}$.

$\mathrm{pHRed}$ 's fluorescence lifetime - the time between excitation and emission - also responds to $\mathrm{pH}$. The authors used this feature in twophoton microscopy to track second-by-second $\mathrm{pH}$ changes in living cells.

A red $\mathrm{pH}$ sensor leaves the often-used green fluorescence wavelength available for simultaneous study of other cellular properties, such as energy metabolism, during multicolour imaging.

J. Am. Chem. Soc. doi:10.1021/ ja202902d (2011)

\section{CANCER BIOLOGY}

\section{When push comes to shove in cancer}

Ovarian cancer cells spread away from the site of a primary tumour by moving healthy cells out of the way, according to work by Joan Brugge of Harvard Medical School in Boston, Massachusetts, and her colleagues.

Brugge's team used timelapse video microscopy to spy on ovarian cancer cells as they interacted with healthy cells in culture in a model of cancer metastasis. When the two cell types came into contact, the extracellular matrix surrounding the healthy cells disassembled, and they moved away from the point of contact with the tumour cells. This displacement, Brugge's group showed, depends on three proteins: $\alpha_{5} \beta_{1}$ integrin, talin 1 and myosin. In addition, the interaction with myosin and talin 1 is required to generate the force that physically pushes healthy cells out of the way.

Cancer Discov. doi:10.1158/ 2159-8274.CD-11-0010 (2011)

\section{OCEAN CHEMISTRY}

\section{Cushion against acidification}

When sulphur and nitrogen gases from ship fuel and other local sources of combustion end up in coastal waters, they strongly diminish ocean acidification by carbon dioxide.

Keith Hunter at the

University of Otago in

Dunedin, New Zealand, and his colleagues used a simple chemical model to compare

COMMUNITY CHOICE

The most viewed papers in science

\title{
BRAIN AND BEHAVIOUR
}

\section{The voter's grey matter}

\section{7 highly ReAd \\ on www.cell.com \\ 15 May-14 June}

The size of certain brain regions offer clues to a person's political leaning, according to Ryota Kanai at University College London and his colleagues.

In brain scans of more than 100 university students, self-reported conservatives had above-average volumes of grey matter in the right amygdala, whereas liberals had more of the grey stuff in the anterior cingulate cortex (ACC). This structural pattern fits with previous work that linked liberalism to high activity in the ACC, which is thought to help the brain sort out conflicting stimuli, as well as research correlating conservatism with higher levels of aggression under threat, an emotion probably processed by the amygdala.

Results aside, perhaps the reason that this paper was so often downloaded is that one of the co-authors is actor Colin Firth. Firth prompted the team to do the study for a BBC Radio 4 programme. Curr. Biol. 21, 677-680 (2011)

the effects of sulphur and nitrogen oxides and ammonia on seawater chemistry with that of $\mathrm{CO}_{2}$. Results from three case studies - in the North Sea, the Baltic Sea and the South China Sea suggest that, after chemical re-equilibration, the net effect of the gases on ocean chemistry is negligible.

The authors conclude that in coastal regions with intense shipping activity, air-sea fluxes of acidic nitrogen and sulphur compounds effectively cancel out expected $\mathrm{CO}_{2}$ induced ocean acidification by buffering reactions involving carbonate and bicarbonate ions in seawater. However, they suspect that such chemical buffering comes at the expense of shutting down much of the ocean uptake of excess atmospheric $\mathrm{CO}_{2}$. Geophys. Res. Lett. doi:10.1029/ 2011GL047720 (2011)

\section{PHYSIOLOGY AND CLIMATE}

\section{Air conditioning all in the nose}

Researchers have long speculated on the benefits offered by the variety of human nose shapes, but few have looked inside the facial feature - or the larger nasal cavity of the skull - for an answer.

Marlijn Noback of Eberhard Karls University in Tübingen, Germany, and her colleagues took computer-

aided measurements of the sizes and shapes of nasal cavities (pictured) in a total of 100 skulls from 10 human groups living in 5 different climates. They found that individuals from cold, dry climates had higher and narrower cavities than those from hot, humid climates. The authors suggest that the high, narrow cavities may help to mix the air and increase its moisture content and temperature as it is inhaled.

Am. J. Phys. Anthropol. doi:10.1002/ajpa.21523 (2011)

\section{$\rightarrow$ NATURE.COM}

For the latest research published by Naturevisit:

www.nature.com/latestresearch 